Andreas Gröschl*, Janik Schaude und Tino Hausotte

\title{
Kontinuierliche laterale Abtastung von Oberflächen durch axiale Regelung des Arbeitsabstandes in einem Nanokoordinatenmessgerät mit einem hochfrequent fokusabstandsmodulierten, fasergekoppelten, konfokalen Punktsensor
}

Lateral closed-loop scans of surfaces using a nano coordinate measuring system and a high-speed focal-distance modulated fibre-coupled confocal sensor

DOI 10.1515/teme-2018-0040

Zusammenfassung: In diesem Artikel wird ein neues Messverfahren zur Oberflächenmessung bei gleichzeitiger axialer Regelung des Arbeitsabstandes eines hochfrequent fokusabstandsmodulierten, fasergekoppelten, konfokalen Punktsensors in einem Nanokoordinatenmessgerät (NMM1) [4] vorgestellt. Während bei diesem Verfahren alle bekannten Vorteile der Konfokaltechnik beibehalten werden, stellt die innovative Kombination einer fasergekoppelten Beleuchtung und Detektion mit einer akustisch getriebenen, modulierbaren Flüssigkeitsgradientenlinse (engl. tunable, acoustically driven gradient-index fluid lens, Abk. TAG-Linse) [10] zur hochfrequenten Abstandmodulation des Fokus zusammen mit einer neuartigen Signalverarbeitung auf Basis einer Lock-in-Verstärkung eine wesentliche Verbesserung dar. Durch die Anordnung und Auswertung ergibt sich im Gegensatz zu konventionellen konfokalen Punktsensoren eine nahezu lineare Kennlinie, wobei die Richtung der Abweichung der Messobjektoberfläche relativ zur Fokusebene des Objektivs eindeutig bestimmt werden kann. Es wird insbesondere die Verwendung des Sensors sowie die Nutzung der resultierenden Kennlinie zur Regelung der Oberflächenposition in einem Nanokoordinatenmessgerät gezeigt. Im Gegensatz zum Verfahren konventioneller Konfokalsensoren, bei der lateral flächenhafte Abtastungen auf vielen diskreten axialen Höhen durchgeführt werden, um daraus für jeden lateralen Messpunkt durch eine Maximalwertsuche einen Höhenwert zu

\footnotetext{
*Korrespondenzautor: Andreas Gröschl, Lehrstuhl für Fertigungsmesstechnik, Friedrich-Alexander-Universität Erlangen-Nürnberg (FAU), E-Mail: andreas.groeschl@fmt.fau.de
}

ermitteln, kann durch die gezeigte Nachregelung eine Oberflächenerfassung durch eine einzige laterale Abstastung erfolgen. Die sich daraus ergebende immense Zeitersparnis ist nicht nur wesentlich ökonomischer sondern weist aufgrund zeitlicher thermischer Driften auch geringere Messabweichungen auf.

Schlüsselwörter: Konfokalsensor, Nanokoordinatenmessgerät, Oberflächenmessung.

Abstract: This article presents measurements of surfaces by performing closed-loop line scans using a nano coordinate measuring system (NMM-1) [4] and a new highfrequency focal-distance-modulated confocal point sensor. While maintaining the known advantages of the confocal measurement, the sensor represents an innovative combination of a fibre-coupled confocal illumination and detection with a tunable, acoustically driven gradient-index fluid lens (TAG-lens) [10] for modulation of the focus distance and a novel signal processing utilizing a lock-in amplifier. The new arrangement is able to achieve an approximately linear characteristic curve for the optimised feedback control of a CMS in scanning sample mode. This article emphasises the implementation and use of the sensor in a coordinate measuring system and the advantages of lateral closed-loop scans holding the distance between the sample and the objective constant compared to conventional lateral scans in axial stepwise layers.

Keywords: Confocal sensor, nano coordinate measuring machine, surface measurement. 


\section{Einleitung}

Die Miniaturisierung und Mikrostrukturierung komplexer Bauteile und die damit einhergehenden immer enger werdenden Fertigungstoleranzen stellen die Messtechnik vor gänzlich neue Herausforderungen. Mit speziellen Mikround Nanopositioniersystemen ist es seit einigen Jahren bereits möglich, hochgenaue, dreidimensionale Relativbewegungen zwischen Sensor und Messobjekt in Bereichen von einigen Millimetern und mit Auflösungen im Nanooder Sub-Nanometerbereich zu realisieren [11]. Der Bedarf an hochgenauen Sensoren zur Antastung der Messobjekte, die solchen herausfordernden Messaufgaben gewachsen sind, ist jedoch nicht gedeckt.

Ein 1957 von Minsky patentiertes und seither bewährtes optisches Verfahren stellt die Konfokalmikroskopie dar [8]. Durch die Kombination einer konfokal angeordneten Beleuchtungs- und Detektionsblende gelangt das von einer Oberfläche reflektierte Licht nur durch die Detektionsblende, wenn sich diese im gemeinsamen Fokus befindet. Dadurch wird eine sehr hohe Tiefendiskrimierung und folglich eine hohe axiale Auflösung ermöglicht. Die Verwendung dieser Konfokaltechnik und deren Einsatz als Punktsensor in konfokalen Laserscanningmikroskopen (engl. confocal laser scanning microscopy, Abk. CLSM) hat damit auch in der Oberflächenmesstechnik einen hohen Stellenwert erlangt [1]. Aufgrund der symmetrisch abfallenden Intensitätsfunktion bei Defokusierung sowie der Abhängigkeit der detektierten Intensität von den Reflexionseigenschaften der Oberfläche muss zur Höhenerfassung jeder laterale Messpunkt mindestens um den Bereich des Maximums dieser Intensitätsfunktion axial abgetastet werden. Anstatt aufeinanderfolgend jeden lateralen Messpunkt axial abzutasten werden häufig auch laterale Abtastungen auf diskreten axialen Höhen durchgeführt, um anschließend für jeden lateralen Punkt die Schicht mit der maximalen Intesität zu ermitteln. Damit ist jedoch eine flächenhafte Erfassung insbesondere von Oberflächen mit hohen axilialen Strukturen sehr zeitaufwendig.

Neben dem Ansatz der axialen Abtastung durch mechanische Verschiebung des Messobjekts oder des Objektivs ist auch die Verwendung adaptiver Optiken zur Modulation der Fokusebene bekannt [6]. Weiterhin werden akustisch getriebene hochfrequent modulierbare Gradientenoptiken in Mikroskopieanwendungen [7] und konfokalen Systemen genutzt [2].

Durch die hier vorgestellte innovative Kombination eines fasergekoppelten, konfokalen Punktsensors mit einer solchen Gradientenoptik zur hochfrequenten Modulation der Fokusposition und einer in diesem Kontext neuartigen
Signalverarbeitung auf Basis einer Lock-in-Verstärkung wird unter Beibehaltung aller bekannten Vorteile der Konfokaltechnik ein Sensor mit einer annähernd linearen Kennlinie geschaffen, der sowohl zur Messung der axialen Höhe als auch zur dynamischen Regelung der axialen Position des Positioniertisches eines Nanokoordinatenmessgerätes genutzt werden kann, um während einer lateralen Abtastung den Arbeitsabstand zwischen Objektiv und Messobjekt konstant zu halten. Dadurch wird es ermöglicht, die zu messende Oberfläche durch eine einzige laterale Abtastung zu erfassen.

\section{Aufbau}

Der im Folgenden dargestellte optische Aufbau sowie die Funktionsweise der Signalentstehung und Signalverarbeitung des beschriebenen Sensors wurden bereits ausführlich in [5] und [3] erläutert und werden zur besseren Nachvollziehbarkeit der im Anschluss illustrierten Messergebnisse hier nochmals kurz zusammengefasst.

Der fokusabstandsmodulierte Konfokalsensor wird durch die in Abbildung 1 rechts dargestellten Komponenten des optischen Systems (1-7) gebildet und könnte als eigenständiges System auch nur mit diesen genutzt werden. Ist die Gradientenoptik (5) ausgeschaltet, kann das System weiterhin auch als konventioneller Konfokalsensor verwendet werden, bei dem keine Fokusmodulation durch die Gradientenoptik erfolgt (s. Abb. 1, durchgezogene rote Linien). Im Detail wird Licht einer monochromatischen Laserquelle (1) in eine Monomodefaser mit Strahlteilung (3) eingekoppelt, am Faserende wieder ausgekoppelt und über eine Kollimationslinse (4) kollimiert. Anschließend passiert der Strahlengang die modulierbare Gradientenoptik (5) sowie den ersten Strahlteiler (8) und wird vom Mikroskopobjektiv in der Fokusebene des Objektivs fokusiert. Das von der zu messenden Oberfläche (7) reflektierte Licht gelangt von dort in umgekehrter Richtung durch die genannten optischen Elemente wieder zum Faserende und wird eben dann eingekoppelt, wenn sich die Messfläche im Fokus befindet. Über den Strahlteiler in der Faser gelangt schließlich ein Teil des eingekoppelten Lichts zum Detektor (2). Durch einen optischen Isolator wird eine Einkopplung des anderen Teils des Lichts in den Laser verhindert, um Leistungsschwankungen zu vermeiden. Wird die modulierbare Gradientenoptik (5) eingeschalten, bewirkt diese eine hochfrequent sinusförmig wechselnde Änderung der Brechkraft und damit eine periodisch ändernde Fokusierung bzw. Aufweitung des zuvor kollimierten Strahls. Die nachfolgende Fokusierung durch ein unendlich korrigiertes 


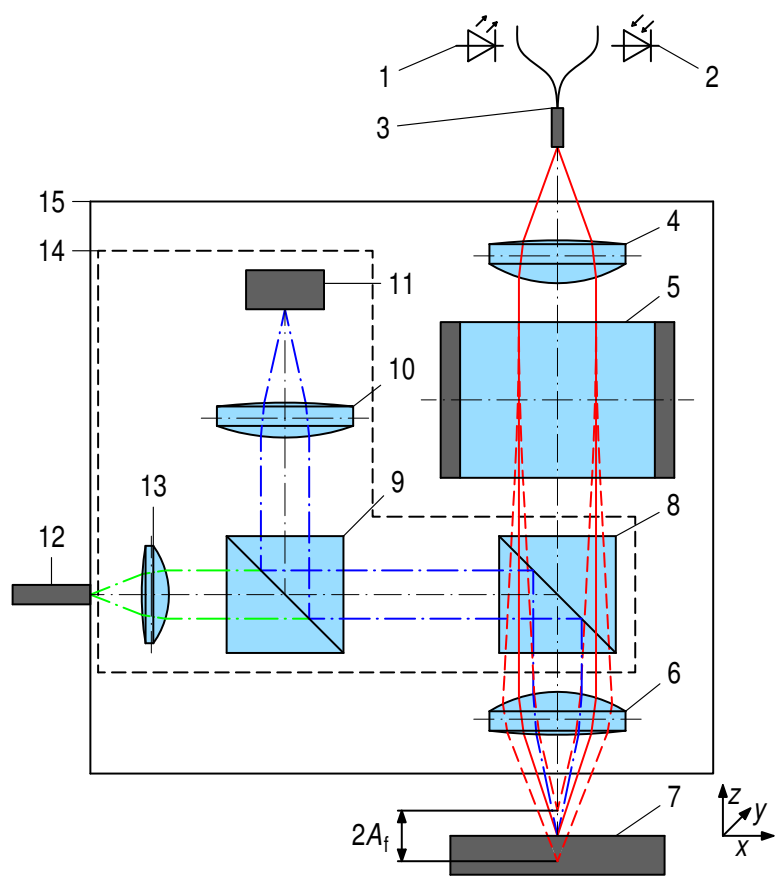

Abb. 1: Optischer Aufbau des fokusabstandsmodulierten Punktsensors mit: 1. Laser, 2. Fotodiode, 3. Lichtwellenleiter mit Strahlteilung, 4. Kollimationslinse, 5. TAG-Linse, 6. Mikroskopobjektiv, 7. Messobjekt, 8. Strahlteiler, 9. Strahlteiler, 10. Tubuslinse, 11. Flächensensor, 12. Weißlichtquelle, 13. Kollimationslinse, 14. bildgebendes System, 15. Sensorkopf, durchgezogene und gestrichelte rote Linien: modulierter Strahlengang der konfokalen monochromatischen Beleuchtung und Detektion, punkt-gestrichelte blaue und grüne Linien: Strahlengang der flächenhaften Erfassung und Beleuchtung in Anlehnung an [4].

Mikroskopobjektiv bewirkt schließlich eine sinusförmige Modulation des fokussierten Laserlichts um die Brennebene des Objektivs mit der Amplitude $A_{\mathrm{f}}$. Wie im folgenden Kapitel gezeigt wird, kann aus dem durch diese Oszillation erzeugten Signal mithilfe einer neuartigen Signalverarbeitung schließlich eine Kennlinie generiert werden, mithilfe derer die Verschiebung der Messfläche zur Brennebene des Objektivs eindeutig bestimmt werden kann.

Die in Abbildung 1 links dargestellten und durch die gestrichelten Linien (14) eingerahmten Komponenten (8-13) dienen der flächenhaften, mikroskopischen Erfassung der Oberfläche. Der von dem Mikroskopobjektiv erfasste Bildbereich wird dazu über zwei Strahlteiler ( $8 \mathrm{u}$. 9) und eine Tubuslinse (10) auf einen Flächensensor (11) abgebildet. Durch eine zusätzliche Beleuchtung (12) und eine Kollimationslinse (13) wird weiterhin eine Auflichtbeleuchtung ermöglicht. Diese flächenhafte Erfassung dient einerseits der lateralen Orientierung auf dem Messobjekt und erleichtert andererseits durch die gleichzeitige Abbildung des Laserfokus die Fokusfindung. Abbildung 2

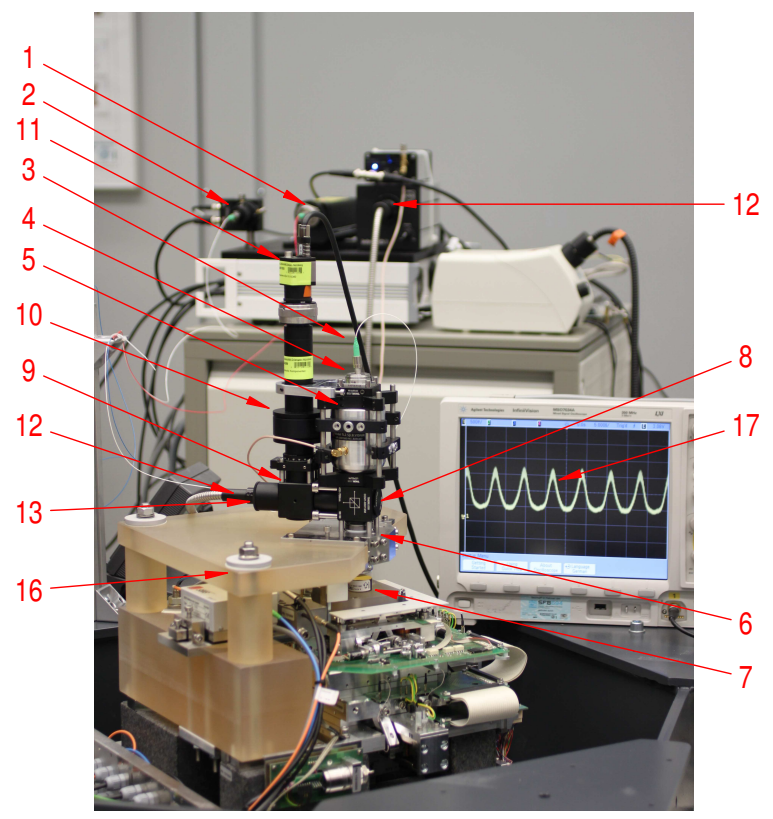

Abb. 2: Technische Realisierung des fokusabstandsmodulierten Punktssensors und Integration in eine Nanokoordinatenmessgerät mit: 1. Laser, 2. Fotodiode, 3. Lichtwellenleiter mit Strahlteilung, 4. Kollimationslinse, 5. TAG-Linse, 6. Mikroskopebjektiv, 7. Messobjekt, 8. Strahlteiler, 9. Strahlteiler, 10. Tubuslinse, 11 Flächensensor, 12. Weißlichtquelle, 13. Kollimationslinse, 16. Nanokoordinatenmessgerät, 17. Fotodiodensignal.

zeigt die technische Realisierung des Sensors sowie dessen Intergration in eine Nanokoordinatenmessgerät (16).

Der hierbei verwendete Helium-Neon-Laser (1) mit einer Wellenlänge von $632,8 \mathrm{~nm}$ besitzt mit $0,8 \mathrm{~mW}$ eine relativ geringe Ausgangsleistung, sodass weder eine Gesundheitsgefährdung durch Streustrahlung noch eine Erwärmung oder Beschädigung des Messobjekts entsteht. Die Modenreinheit der transversal-elektromagnetischen Mode $\mathrm{TEM}_{00}$ beträgt mehr als $95 \%$. Das Licht dieser Mode mit einem Durchmesser von 0,48 mm wird über einen optischen Isolator mithilfe eines Kollimators mit einer effektiven Brennweite von $2 \mathrm{~mm}$ und einer numerischen Apertur von 0,5, bei guter Justierung mit einem Wirkungsgrad von über $90 \%$ in eine Singlemodefaser mit einem Kerndurchmesser von 3,5 $\mu \mathrm{m}$ eingekoppelt. Der Lichtwellenleiter (LWL) ist über einen FC-APC Stecker mit einem Schrägschliff mit dem Kollimator verbunden, um Rückreflexionen am Faserende zu minimieren. Weiterhin ist im LWL ein Strahlteiler (3) mit einem Teilungsverhältnis von 50:50 integriert, sodass das zweite Faserende mit einer Avalanche-Fotodiode (2) verbunden werden kann. Der Detektor verfügt über eine einstellbare Verstärkung, wobei je nach Verstärkungsfaktor eine Sättigung zwischen $8 \mu \mathrm{W}$ bis $80 \mu \mathrm{W}$ bei $600 \mathrm{~nm}$ eintritt. Unter der Annahme verlustfreier Ein- und Auskopplungen wird die Laserleis- 
tung mit dem weiteren Strahlteiler (8) mit dem gleichen Teilungsverhältnis von 50:50 ingesamt viermal halbiert, sodass eine maximale Leistung von $0,8 \mathrm{~mW} / 16=50 \mu \mathrm{W}$ am Detektor anliegen könnte. Durch Kopplungverluste an den Kollimatoren und dem Objektiv sowie schlechter Reflexionseigenschaften der zu messenden Oberfläche, kann die detektierte Leistung jedoch entsprechend deutlich geringer ausfallen. Der Kollimator (4) mit einer effektiven Brennweite von $11 \mathrm{~mm}$ erzeugt einen kollimierten Strahldurchmesser von 1,8 mm. Dieser wurde so gewählt, dass die Öffnungspupille des verwendeten 100x Mikroskopobjektivs mit einem Arbeitsabstand von 4,5 mm gerade ausgeleuchtet wird. Zur bildhaften Darstellung wird ein monochromatischer Sensor mit einer Auflösung von 2048 $\times 1088$ Pixel $(2,23 \mathrm{MPixel})$ und einer maximalen Bildrate von 152 Bildern pro Sekunde genutzt. Die Abbildung erfolgt hierbei über eine $200 \mathrm{~mm}$ Tubuslinse. Die LEDWeißlichtquelle (12) hat eine Farbtemperatur von $6000 \mathrm{~K}$ mit einem maximalen Lichtstrom am Lichtleiteraustritt von 500 Lumen.

\section{Signalentstehung und -verarbeitung}

Die Grundlagen der paraxialen Optik wurden 1977 von Sheppard und Choudhury geschaffen [9]. Weiterhin wurde 1984 durch Wilson and Sheppard [12] gezeigt, dass die axiale Verteilung der normierten Intensitätsfunktion, welche an einem idealen Konfokalsensor mit infinitesimal kleiner Beleuchtungs- und Detektionsblende und einer Ebene als Reflektor bei axialer Verschiebung dieser entsteht, wie folgt beschrieben werden kann:

$$
I(u)=\frac{\sin ^{2} u / 2}{(u / 2)^{2}},
$$

mit der normierten optischen axialen Koordinate

$$
u=8 \pi \lambda z \sin ^{2}(\alpha / 2)
$$

sowie dem halben Öffnungswinkel des Objektivs

$$
\alpha=\arcsin (\mathrm{NA}),
$$

wobei $z$ die relative Verschiebung des ebenen Reflektors zur Fokusebene darstellt. Für ein 100x Objektiv mit großem Arbeitsabstand und einer numerischen Apertur NA=0,8 ergibt sich damit der in Abbildung 3 gestrichelt dargestellte theoretische Intesitätsverlauf in Abhängigkeit der axialen Verschiebung $z$ mit einer Halbwertsbreite (engl. Full Width at Half Maximum, Abk. FWHM) von $0,7 \mu \mathrm{m}$. Im

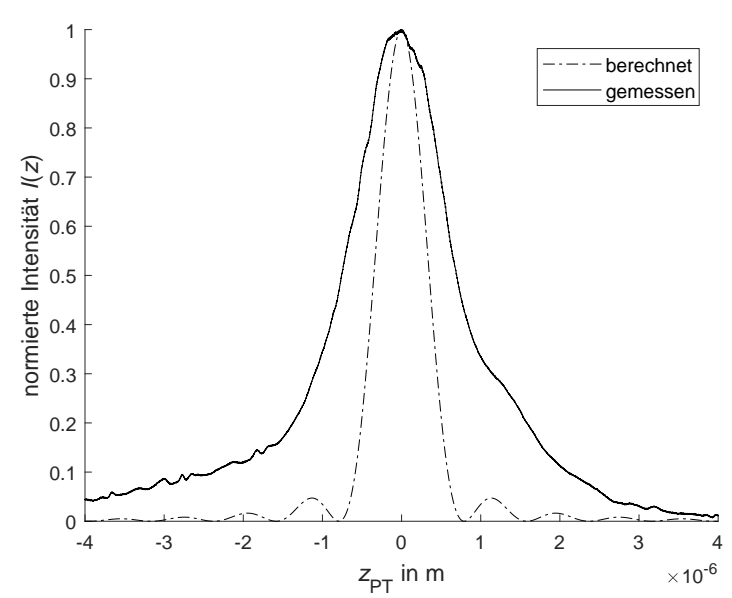

Abb. 3: Normierte detektierte (durchgezogene Linie) Intensität und simulierte Intensität (gestrichelt) mit jeweils einem 100x Mikroskopobjektiv mit NA=0.8 ohne Modulation der Gradientenoptik über die axiale Position des Positioniertisches $z_{\mathrm{PT}}$ nach [4].

Vergleich dazu wurde mit dem oben beschriebenen Sensor bei ausgeschalteter Gradientenoptik der Intensitätsverlauf in Abhängigkeit der axialen Position $z_{\mathrm{PT}}$ des Positioniertisches gemessen und normiert (s. durchgezogene Linie). Aufgrund der finiten Größe der verwendeten Faser, welche in dem gezeigten System sowohl als Beleuchtungs- als auch als Detektionsblende fungiert, ist die gemessene axiale Intensitätsfunktion mit einer Halbwertsbreite von 1,5 $\mu \mathrm{m}$ naturgemäß deutlich breiter als die eines idealen konfokalen Systems mit infinitesimal kleinen Blenden, wie bei Vergleich der beiden Kurven ersichtlich wird. In konventionellen Konfokalmikroskopen wird zur Ermittlung der Oberfläche das Maximum dieser axialen Intensitätsfunktion bestimmt. Dazu muss jeder laterale Messpunkt axial abgetastet werden, um den gezeigten Intensitätsverlauf zu ermitteln. Statt der mechanischen Verschiebung der Oberfläche entlang der optischen Achse erfolgt die Abtastung in dem hier vorgestellten Sensor durch die hochfrequente, sinusförmige Modulation der Fokusposition mithilfe der akkustisch getriebenen Gradientenoptik. Die relative Fokusposition des Linsensystems aus Gradientenoptik und Mikroskopobjektiv zur Brennebene des Objektivs lässt sich daher wie folgt beschreiben:

$$
z_{\text {Fokus }}=A_{\mathrm{f}} \sin \left(2 \pi t f_{\mathrm{TAG}}\right),
$$

mit der Modulationsfrequenz der Gradientenoptik $f_{\mathrm{TAG}}$, Fokusmodulationsamplitude $A_{\mathrm{f}}$ und der Zeit $t$. Bei kontinierlicher Verschiebung des Positioniertisches mit der Geschwindigkeit $v_{\mathrm{PT}}$ lässt sich weiterhin die relative Position des Positioniertisches zur Fokusebene des Objektivs 
beschreiben durch:

$$
z_{\mathrm{PT}}(t)=v_{\mathrm{PT}} \cdot t
$$

Damit ist die relative Position der Messfläche zur modulierten Fokusposition die Differenz zwischen der Tischposition und der modulierten Fokusposition gemäß:

$$
z(t)=v_{\mathrm{PT}} \cdot t-A_{\mathrm{f}} \sin \left(2 \pi t f_{\mathrm{TAG}}\right) .
$$

Für eine hypothetische Verfahrgeschwindigkeit von $v_{\mathrm{PT}}=$ $0,04 \mathrm{~m} / \mathrm{s}$ und der ersten Resonanzfrequenz der Gradientenoptik ergeben sich damit die in Abbildung 4 gezeigten Verläufe für $z$ und $z_{\mathrm{PT}}$, wobei die vertikalen roten Linien die Positionen kennzeichnen, an denen der Fokus auf der Messfläche liegt. Diese hier simulierte Geschwindigkeit dient lediglich der Veranschaulichung der Signalentstehung und Signalverarbeitung und liegt bei der Verwendung des Nanokoordinatenmessgeräts im Bereich weniger $\mu \mathrm{m} / \mathrm{s}$.

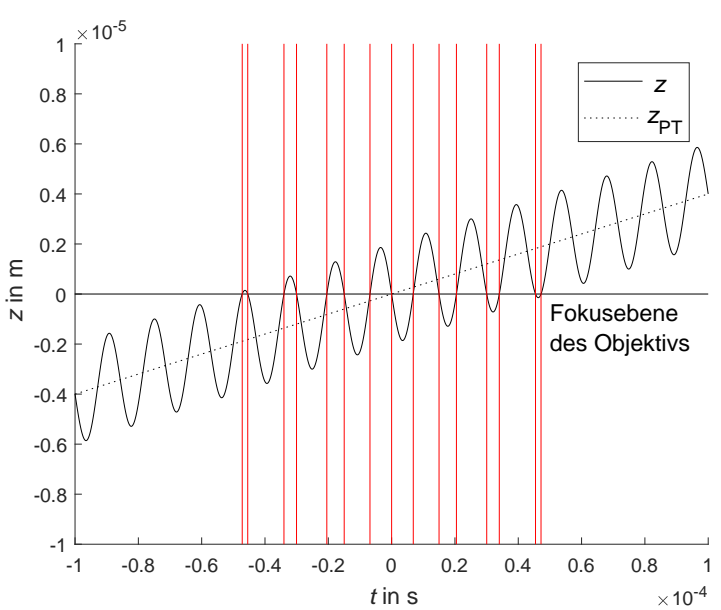

Abb. 4: Relative Position der Messfläche zur modulierten Fokusposition $z$ (durchgezogen) und relative Position der Messfläche zur Fokusebene des Objektivs $z_{\mathrm{PT}}$ (gestrichelt) über die Zeit $t$ nach [4].

Durch Substitution der Gleichungen 2 und 3 in Gleichung 1 und Einsetzen der Werte aus Abbildung 4 bzw. Gleichung 6 ergibt sich die in Abbildung 5 dargestellte normierte Intensität $I$ in Abhängigkeit der Zeit $t$. Ana$\log$ zu Abbildung 4 kennzeichnen die vertikalen roten Linien die Zeitpunkte der Intensitätsmaxima zu denen die modulierte Fokusposition des Linsensystems mit der Messobjektoberfläche übereinstimmt.

Hieraus wird ersichtlich, dass sich durch den Abstand der Maxima respektive durch die daraus resultierende charakteristische Signalform mithilfe einer geeignete Signalverarbeitung eindeutig der relative Abstand des Messobjektes zur Fokusebene des Objektivs ermitteln lässt. Theoretisch

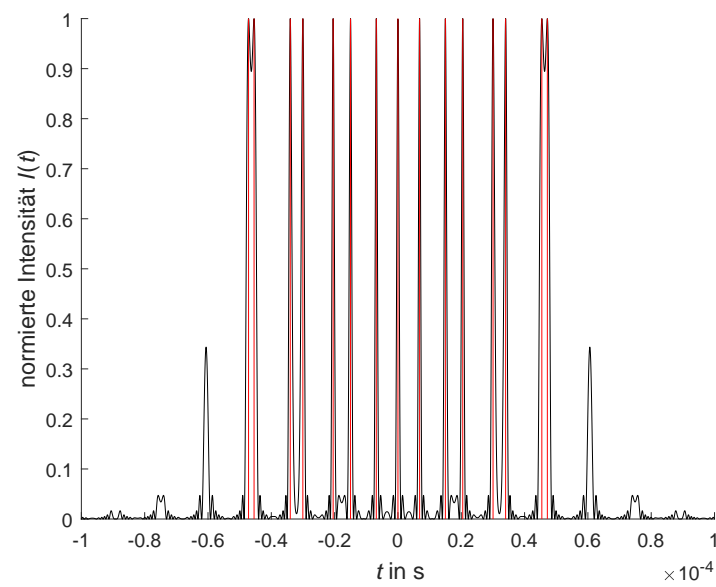

Abb. 5: Normierte simulierte Intensität mit einem 100x Mikroskopobjektiv mit NA $=0.8$ und Fokusmodulation durch die Gradientenoptik über die Zeit $t$ bei einer Abtastgeschwindigkeit $v_{\mathrm{PT}}=0,04 \mathrm{~m} / \mathrm{s}$ und einer Fokusmodulationsamplitude $A_{\mathrm{f}}= \pm 2 \mu \mathrm{m}$ nach [4]

wären hierfür bimodale Gaußregressionen möglich, um den zeitlichen Abstand der Maxima zu ermitteln. Eine besonders innovative Signalverarbeitung, welche bereits in [5] gezeigt und in [3] mithilfe einer eigenen Field Programmable Gate Array (FPGA)-basierten Signalverarbeitung umgesetzt wurde, beruht auf dem Prinzip der Lock-in-Verstärkung. Allgemein stellt diese eine mit dem Verstärkungsfaktor $S$ multiplizierte Mittelwertbildung der Integration über ein Vielfaches $n$ der Periodendauer $T$ des multiplizierten Eingangssignals $V_{\mathrm{E}}$ mit einem Referenzsignal $V_{\mathrm{R}}$ dar. Damit kann das Ausgangssignal wie folgt berechnet werden:

$$
V_{\mathrm{A}, k}\left(t^{*}\right)=\frac{S}{n T} \int_{t^{*}-n T}^{t^{*}} V_{\mathrm{E}}(t) \cdot V_{\mathrm{R}, k}(t) \mathrm{d} t \quad n \in \mathbb{N}
$$

mit dem Referenzsignal

$$
V_{\mathrm{R}, k}(t)=\sin \left(2 \pi \cdot t \cdot f_{\mathrm{ref}, k}+\phi\right),
$$

wobei die Referenzfrequenz $f_{\text {ref }, k}$ ein Vielfaches $k$, auch als $k$-te harmonische Referenzfrequenz bezeichnet, der Modulationsfrequenz der Gradientenoptik darstellt:

$$
f_{\text {ref }, k}=k \cdot f_{\mathrm{TAG}} \quad k \in \mathbb{N} .
$$

Weiterhin kann ein Mittelwertsignal des Eingangsignals gemäß:

$$
V_{\mathrm{M}}\left(t^{*}\right)=\frac{S}{n T} \int_{t^{*}-n T}^{t^{*}} V_{\mathrm{E}}(t) \mathrm{d} t \quad n \in \mathbb{N}
$$


gebildet werden. Wie aus den Gleichungen (7) und (10) ersichtlich wird, sind diese nicht nur von der Form, sondern auch von der Amplitude des Eingangssignals $V_{\mathrm{E}}$ abhängig, sodass unterschiedliche Reflexionseigenschaften verschiedener Messobjekte zu Verstärkungs- und Linearitätsabweichungen führen würden. Unter der Annahme einer während der Integrationszeit $n T$ konstant bleibenden Amplitude des Eingangssignals kann eine Normierung durch die Quotientenbildung des 3. harmonischen Ausgangssignals $V_{\mathrm{A}, 3}$ und dem 2. harmonischen Ausgangssignals $V_{\mathrm{A}, 2}$ generiert werden, um eine von den Reflexionseigenschaften der Messobjektoberfläche unabhängige Kennlinie zu bilden, wie in [5] und [3] gezeigt wurde und in Abbildung 6 dargestellt ist. Weiterhin kann der Quotient aus dem 2. harmonischen Ausgangssignal $V_{\mathrm{A}, 2}$ und dem Mittelwertsignal $V_{\mathrm{M}}$ genutzt werden, um über einen Schwellwert $S W$ den unteren und oberen Kennlinienbereich $K_{\mathrm{UG}}$ und $K_{\mathrm{OG}}$ ebenfalls unabhängig von den Reflexionseigenschaften des Messobjektes zu definieren.

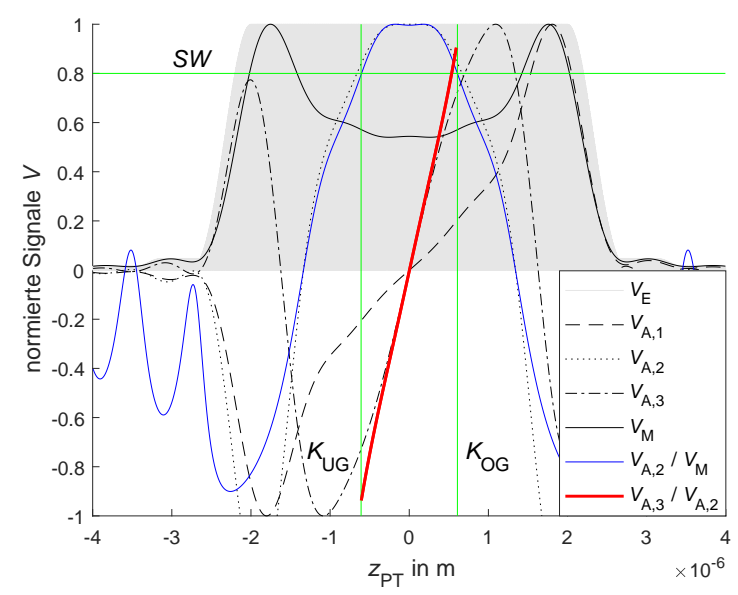

Abb. 6: Simulierte Ausgangssignale mit Lock-in-Verstärkung auf einem planaren idealen Messobjekt.

\section{Messergebnisse und Auswertung}

Um die Messpräzision des neu entwickelten Sensors zu evaluieren, wurden mit diesem auf einem Raunormal wiederholt 25 Messungen durchgeführt, wobei die annähernd lineare Kennlinie zur axialen Regelung des Arbeitsabstandes genutzt wurde. Die z-Koordinaten des Messobjekts $z_{\mathrm{MO}}$ lassen sich errechnen, indem zur negierten axialen Position des Positioniertisches die mittels der Kennlinie errechnete Regelabweichung, welche maximal 148,3 nm beträgt, addiert wird. In Abbildung 7 sind die so erzielten Ergebnisse dargestellt. Der laterale Abtastbereich beträgt $30 \mu \mathrm{m}$ und die Abtastgeschwindigkeit $2 \mu \mathrm{m} / \mathrm{s}$, sodass eine Messung innerhalb von $15 \mathrm{~s}$ abgeschlossen ist. Hierbei wird alle $5 \mathrm{~nm}$ ein Messpunkt aufgenommen. Wie aus Abbil-

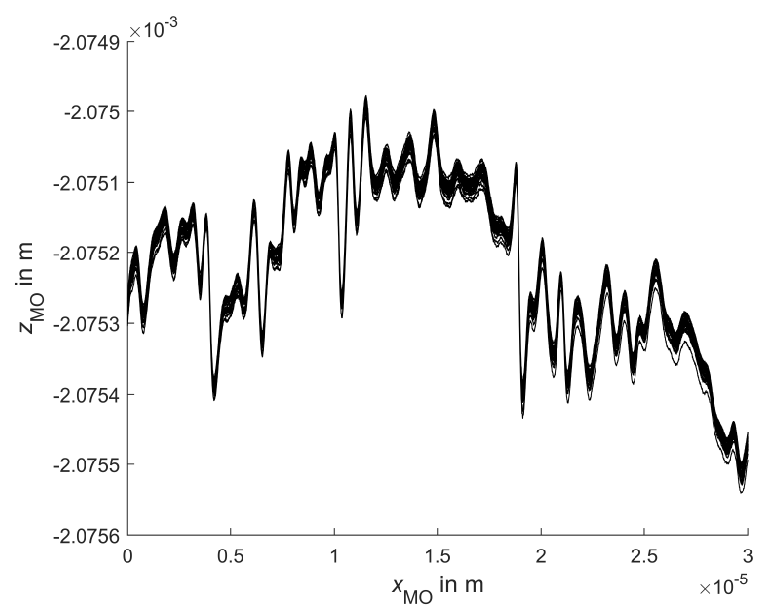

Abb. 7: 25 wiederholte Scanmessungen mit axialer Nachregelung.

dung 7 ersichtlich wird, weichen die einzelnen Messergebnisse voneinander ab. Die maximale Spannweite über alle $\mathrm{x}$-Werte liegt bei $53,1 \mathrm{~nm}$. Wie in [5] für diesen Messaufbau bereits gezeigt, stellen die Temperaturschwankungen einen wesentlichen Einflussfaktor auf das Messergebnis dar, da diese eine Drift der Messergebnisse verursachen. Um diese herauszurechnen, wird für jede Messung der Mittelwert von $z_{\mathrm{MO}}$ gebildet und von den einzelnen Werten subtrahiert. In Abbildung 8 sind die so korrigierten Messergebnisse grafisch dargestellt. Da für wiederholte Messungen die $\mathrm{x}$-Werte, an welchen die Messpunkte aufgenommen werden, nicht exakt identisch sind, werden alle erfassten Werte auf ein einheitliches Gitter an x-Werten mit dem Gitterabstand $5 \mathrm{~nm}$ linear interpoliert. Dabei beträgt die maximale Spannweite über alle $\mathrm{x}$-Werte $33,3 \mathrm{~nm}$, die maximale Standardabweichung $7,8 \mathrm{~nm}$ und die über alle x-Werte gemittelte Standardabweichung 3,2 nm.

An derselben Stelle wurden ebenfalls nach dem Verfahren der konventionellen Konfokalmikroskopie 25 wiederholte Messungen durchgeführt, wobei derselbe optische Aufbau mit ausgeschalteter TAG-Linse verwendet wurde. Pro Messung werden 124 laterale Abtastungen auf unterschiedlichen axialen Höhen durchgeführt. Die Höhendifferenz zwischen zwei aufeinanderfolgenden Abtastungen beträgt $85 \mathrm{~nm}$, die Messdauer für eine komplette Messung ca. 35 Minuten. Die Höhe des Messobjekts an 


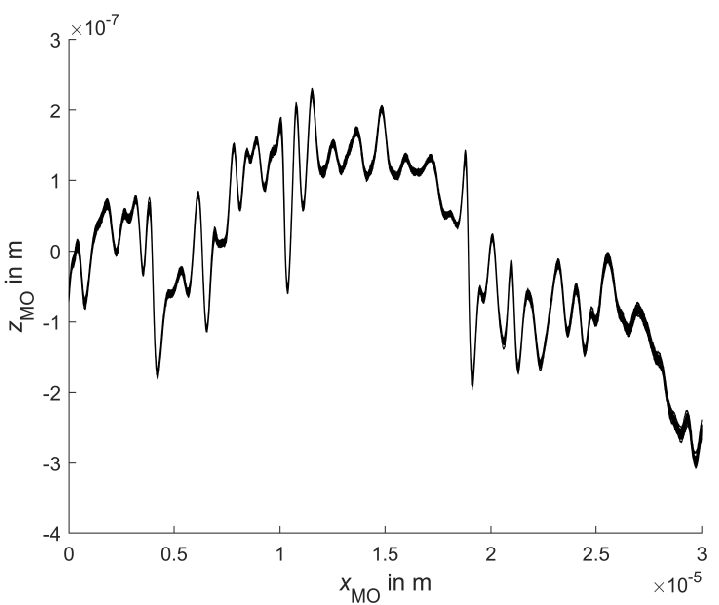

Abb. 8: 25 wiederholte Scanmessungen mit axialer Nachregelung und Driftkorrektur.

jedem $\mathrm{x}$-Wert wird bestimmt, indem für jeden einzeln die Position des Maximums einer gefitteten Gauß-Kurve ermittelt wird. Bei den so erhaltenen Messwerten liegt die maximale Spannweite ohne Driftkorrektur bei $57,6 \mathrm{~nm}$ und damit nur unwesentlich höher als bei dem neuen Messverfahren. Da die Messungen in einem auf $\pm 0,2{ }^{\circ} \mathrm{C}$ geregelten Messraum stattfinden, sind wesentlich größere Temperaturdriften auch nicht zu erwarten. Allerdings lässt sich diese Spannweite mit der Driftkorrektur nur auf $48,7 \mathrm{~nm}$ reduzieren, was wesentlich mehr ist als bei der neuen Messmethode, da sich Temperaturschwankungen zwischen einzelnen Scans nicht korrigieren lassen, weshalb die längere Messdauer zu erhöhten Abweichungen führt. Dies spiegelt sich auch in erhöhten Standardabweichungen für jeden $\mathrm{x}$-Wert wider, sodass diese im Mittel 3,9 nm beträgt und einen maximalen Wert von 11,3 nm annimmt.

\section{Fazit}

Es konnte gezeigt werden, dass durch den neu entwickelten Sensor eine kontinuierliche laterale Abtastung von Oberflächen durch axiale Regelung des Arbeitsabstandes möglich ist. Im Vergleich zur konventionellen Konfokalmikroskopie reduziert sich hierdurch die Messdauer drastisch, im gezeigten Fall mit relativ geringer axialer Höhe des Messobjekts um 99,3\%, respektive erhöht sich die Messpräzision. Da beide Messmethoden mit dem identischen optischen Aufbau durchgeführt wurden, sind diese Verbesserungen nicht auf eine Änderung desselben zurückzuführen. Gerade bei der Messung unbekannter Topografien bietet die Möglichkeit der Nachregelung einen immensen zeitlichen Vorteil, da mit konventionellen konfokalen Punktsenso- ren bei der ersten Messung aufgrund der Unbekanntheit der Lage der Messobjektoberfläche größtenteils Bereiche gemessen werden, in welchen sich das Messobjekt nicht befindet. Somit bringt die Anwendung des neuen Sensors selbst dann Vorteile, wenn unbekannte Topografien mit der konventionellen Konfokalmikroskopie gemessen werden sollen, da sich mit dem neuen Sensor in einer ersten Messung die Lage der Topografie ermitteln lässt.

Danksagung: Die Autoren möchten der Deutschen Forschungsgemeinschaft (DFG) für die Finanzierung des Projekts (HA 5915/10-1) danken.

\section{Literatur}

[1] C. Cremer und T. Cremer. Considerations on a laser-scanningmicroscope with high resolution and depth of field. Microsc. Acta, 81(1):31-44, 1978.

[2] M. Duocastella, G. Vicidomini und A. Diaspro. Simultaneous multiplane confocal microscopy using acoustic tunable lenses. Optics Express, 22(16):19293-19301, 2014.

[3] A. Gröschl, S. Köhnen, J. Schaude und T. Hausotte. Improvements of a high-speed focus distance modulated fibrecoupled confocal sensor for nanocoordinate measuring systems. 19 . ITG-/GMA-Fachtagung, 2018.

[4] T. Hausotte, B. Percle und G. Jäger. Advanced threedimensional scan methods in the nanopositioning and nanomeasuring machine. Meas. Sci. Technol., 20(8):1-8, 2009.

[5] T. Hausotte, A. Gröschl und J. Schaude. High-speed focaldistance-modulated fiber-coupled confocal sensor for coordinate measuring systems. Appl Opt., 57(14):3907-3914, 2018.

[6] N. Koukourakis, M. Finkeldey, M. Stürmer, C. Leithold, N. C. Gerhardt, M. R. Hofmann, U. Wallrabe, J. W. Czarske und A. Fischer. Axial scanning in confocal microscopy employing adaptive lenses (cal). Optics Express, 22(5): 6025-6039, 2014.

[7] A. Mermillod-Blondin, E. Mcleod und C. B. Arnold. Highspeed varifocal imaging with a tunable acoustic gradient index of refraction lens. Optics Express, 33(18):2146-2148, 2008.

[8] M. Minsky. Memoir on inventing the confocal scanning microscope. Scanning, 10:128-138, 1988.

[9] C. Sheppard und A. Choudhury. Image formation in scanning microscope. Opt. Acta, 24:1051-1073, 1977.

[10] Tag Optics Inc. The physics behind tag optics' technology and the mechanism of action of using sound to shape light. Datasheet, 2013.

[11] E. Uhlmann, B. Mullany, D. Biermann, K. P. Rajurkar, T. Hausotte und E. Brinksmeier. Process chains for highprecision components with micro-scale features. CIRP Annals - Manufacturing Technology, 65:549-572, 2016.

[12] T. Wilson und C. Sheppard. Theory and Practice of Scanning Optical Microscopy. Academic Press, 1984. ISBN 0-12757760-2. 